\title{
Bocio del mediastino posterior - abordaje mínimamente invasivo: videotoracoscopia derecha más cervicotomía ${ }^{\square}$
}

\author{
Intrathoracic goiter resection by combined surgical approach: \\ resection by right videothoracoscopy and cervicotomy
}

\section{David Rodríguez-Ramos', Verónica Acevedo-Mejía² Linkedin, Ricardo Zapata- González ${ }^{3}$ linkedin, Luis Bolívar-Mendoza ${ }^{3}$, Carlos Saldarriaga-Henao ${ }^{3}$}

Puede visualizar el video que acompaña el artículo

Fecha correspondencia: Recibido: octubre 28 de 2015. Revisado: julio 21 de 2016. Aceptado: julio 21 de 2016.

\section{Forma de citar} Rodríguez-Ramos D, Acevedo-Mejí V, Zapata-González R, BolívarMendoza L, Saldarriaga-Hena C. Bocio del mediastino posterior - abordaje mínimamente invasivo: videotoracoscopia derecha más cervicotomía. Rev CES Med 2016. 30(2): 210-216.

\section{Open access}

(C) Copyriaht

Licencia creative commons

Ética de publicaciones

Revisión por pares

Gestión por Open Journal System

ISSN 0120-8705

e-ISSN 2215-9177

Comparte

\section{Resumen}

La cirugía es el manejo recomendado para el bocio intratorácico y cerca de un $90 \%$ de ellos pueden resecarse mediante cervicotomía. El bocio en el mediastino posterior ha sido manejado satisfactoriamente empleando diversos abordajes cervicotorácicos, existiendo poca información en la literatura acerca del uso de la videotoracoscopia como parte de estas técnicas combinadas.

Presentamos el caso de un paciente con un bocio intratorácico con extensión a mediastino posterior, en quien, dadas las características de la masa y su localización, sumado a las ventajas ampliamente conocidas de la cirugía mínimamente invasiva, se decide remplazar la toracotomía y la esternotomía por la videotoracoscopia, permitiendo una buena exposición y disección segura del mediastino.

Consideramos que la videotoracoscopia es una técnica segura y efectiva en casos seleccionados de pacientes con bocio con extensión a mediastino posterior.

Palabras clave: Bocio intratoracico, Bocio mediastino posterior, Videotoracoscopia, Toracotomía, Esternotomía.

\section{Abstract \\ Surgery is the recommended treatment for intrathoracic goiter. About $90 \%$ of the lessons can be resected by cervicotomy. The posterior mediastinal goiter was been successfully managed using different cervico-thoracic approaches; however, there is little available information in the literature about the use of videothoracoscopy as part of these combined techniques. We present a case of a patient with a intrathoracic goiter extended to the posterior mediastinum, in which, given the characteristics of the mass and its location added to the widely known benefits of minimally invasive sur- gery, it was decided to replace thoracotomy and sternotomy with videotho- racoscopy allowing good exposure and safe dissection of the mediastinum.}


Julio - Diciembre 2016 - Pág 211

\section{Sobre los autores:}

1. Residente de IV año de Cirugía General, Universidad CES,

Medellín, Colombia.

2. Cirujana General, Universidad CES, Medellín, Colombia.

Servicio de Cirugía Torácica, Clínica CardioVID - Fundación Cardiovascular Santa María. Medellín, Colombia

3. Cirujanos de tórax y trasplante pulmonar. Servicio de cirugía torácica, Clínica CardioVID Fundación Cardiovascular Santa María. Medellín, Colombia.
We consider that videothoracoscopy is a safe and effective technique in selected cases of patients with goiter extending into the posterior mediastinum.

Keywords: Intrathoracic goiter, Posterior mediastinum goiter, Videothoracoscopy, Thoracotomy, Sternotomy.

\section{Introducción}

El bocio intratorácico tiene una incidencia hasta del 50 \% dependiendo de la definición utilizada y según su dependencia de la glándula tiroides se clasifica en primario y secundario. El bocio primario representa el $1 \%$ de todos los bocios intratorácicos y se caracteriza por ser independiente de la glándula tiroidea cervical. El bocio secundario representa el $99 \%$ restante y se debe a un crecimiento de la glándula tiroides hacia el mediastino, ya sea hacia el mediastino medio o más rara vez hacia el mediastino posterior (1- $\underline{3})$. El bocio en el mediastino posterior representa hasta un $15 \%$ de todos los bocios intratorácicos $(\underline{4}, \underline{5})$.

Existen múltiples estrategias de tratamiento, tanto quirúrgicas como no quirúrgicas. El abordaje quirúrgico es el recomendado, pues permite el diagnóstico histológico además de proporcionar un alivio rápido a los síntomas $(\underline{1}, \underline{2}, \underline{5})$. Más del $90 \%$ los bocios intratorácicos pueden ser resecados por cervicotomía. El abordaje cervicotorácico se indica en casos de bocio intratorácico primario o bocios con extensión al mediastino posterior. Los abordajes combinados de cervicotomía más esternotomía o cervicotomía más toracotomía son los más mencionados en la literatura (4, $, 6,7)$.

La videotoracoscopia en combinación con la cervicotomía, aún permanece como una técnica poco explorada en el manejo de los bocios intratorácicos.

Se presenta el caso de un paciente con bocio intratorácico secundario con extensión a mediastino posterior, resecado exitosamente por videotoracoscopia derecha más cervicotomía. Se realiza una revisión retrospectiva de un caso clínico. Se contó con la aprobación del comité de ética de la institución y del paciente.

\section{Caso clínico}

Se trata de un paciente de 50 años con un cuadro clínico de tres meses de evolución de disnea progresiva, sibilancias, tos matutina, dolor retroesternal y estridor.

La videotoracoscopia en combinación con la cervicotomía, aún permanece como una técnica poco explorada en el manejo de los bocios intratorácicos.
El paciente se presentó en el servicio de urgencias por una exacerbación súbita de su dolor. En el electrocardiograma se evidenciaba una elevación transitoria del segmento ST y en la radiografía de tórax se apreciaba ensanchamiento del mediastino.

Se realizó coronariografía que descartó enfermedad coronaria y posteriormente, en una tomografía cervicotorácica, se encontró una glándula tiroides aumentada de tamaño, heterogénea, con un lóbulo tiroideo derecho de $8 \times 8 \times 12 \mathrm{~cm}$, que se extendía inferiormente hacia mediastino medio y región posterior, estando en contacto con la cara latero posterior de la tráquea, con la cara lateral del tercio medio del esófago y con la cara posterior de la vena innominada hasta el nacimiento del bronquio principal derecho, la cual era sugestiva de un bocio intratorácico posterior (figuras 1 -2).

Dado el tamaño de la masa y la sintomatología del paciente se propuso realizar manejo quirúrgico por técnica combinada de videotoracoscopia derecha más cervi- 


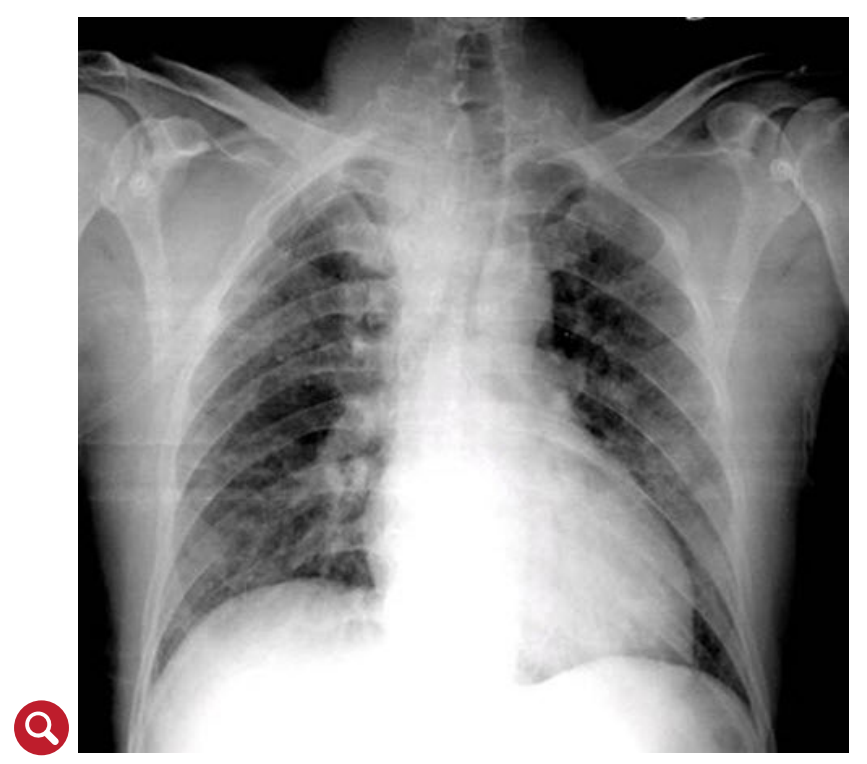

Figura 1. Imágenes prequirúrgicas. Rx de tórax que evidencia ensanchamiento mediastinal.

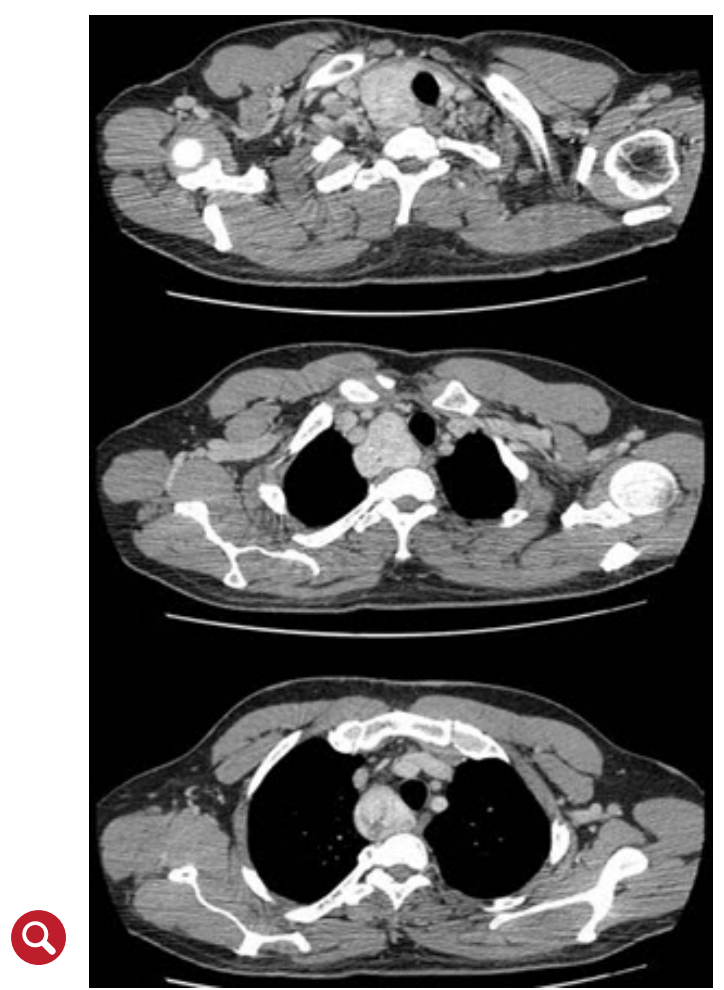

cotomía. Bajo anestesia general y ventilación selectiva unipulmonar izquierda, en decúbito supino con inclinación lateral izquierda de $30^{\circ}$, se realizó videotoracoscopia derecha por tres puertos, lográndose la disección y liberación de la masa de las estructuras del mediastino medio y posterior (figura 3).

Por cervicotomía transversa supra-esternal se completa la disección y extracción de la masa, realizándose hemitiroidectomía derecha.

Figura 2. Secuencia de la tomografía axial computarizada. Se observa masa cervical extendiéndose a mediastino posterior

El postoperatorio del paciente cursó sin complicaciones, retirándose el drenaje pleural el segundo día postoperaotorio, y dando de alta al paciente en el quinto día luego del retiro del dren cervico-mediastinal. En el postoperatorio el paciente presentó un dolor máximo de $3 / 10$, según la escala análoga del dolor, no presentando atelectasias ni complicaciones respiratorias. Las revisiones a los 30 días y a los tres meses fueron normales, con desaparición de los síntomas y regresando a una clase funcional l. 
Julio - Diciembre 2016 - Pág 213

La cervicotomía es la vía de abordaje más común, siendo en el 90 $\%$ de los casos suficiente para lograr la resección completa del bocio y requiriendo en el 10 $\%$ restante otros abordajes ya sean aislados o en combinación con la cervicotomía.
El resultado de la anatomía patológica correspondió a una hiperplasia nodular adenomatosa tipo bocio multinodular.

\section{Discusión}

En aproximadamente el 50 \% de los casos la histología del bocio corresponde a un bocio multinodular (ㅇ). Con respecto al manejo quirúrgico, la cervicotomía es la vía de abordaje más común, siendo en el 90 \% de los casos suficiente para lograr la

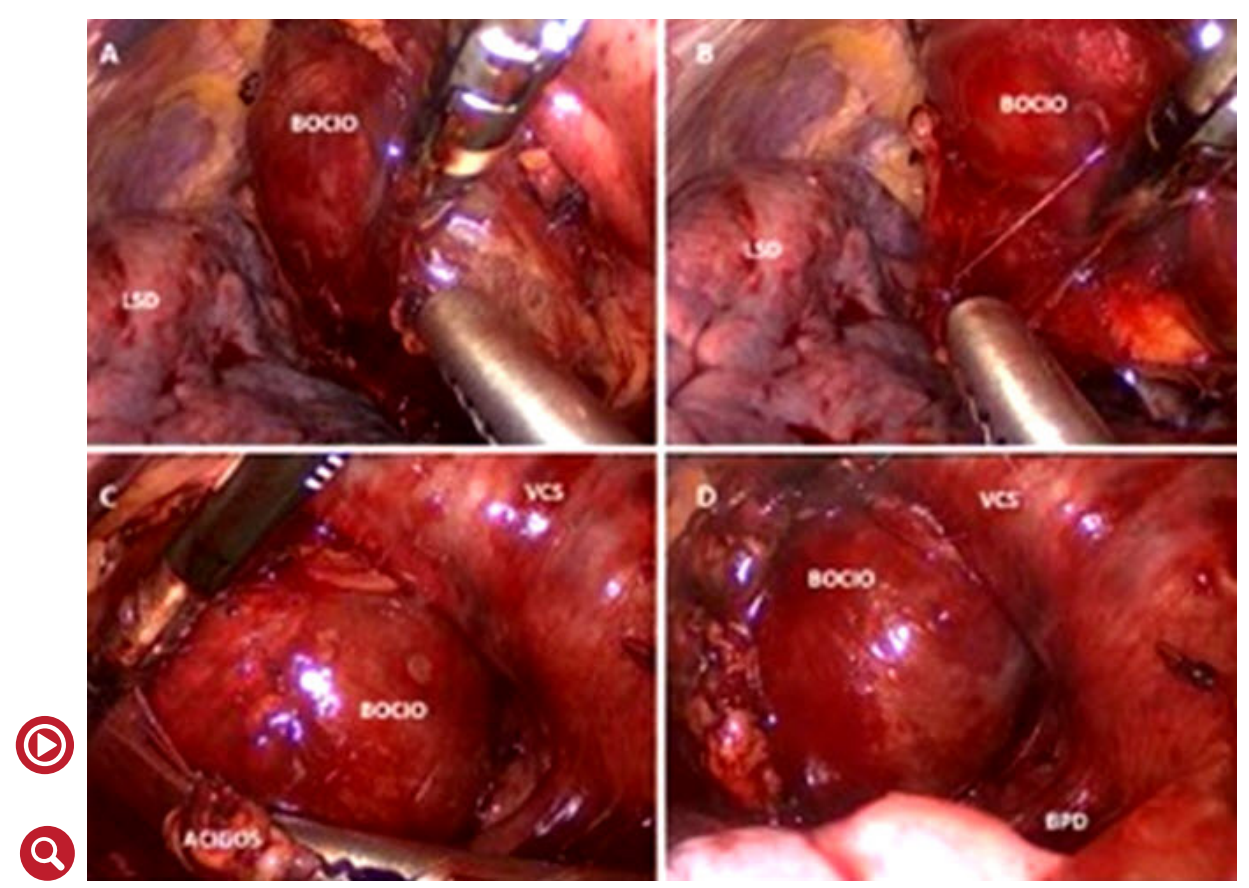

Figura 3. Disección por videotoracoscopia del bocio en el mediastino. A y B. Apertura de la pleura mediastinal. C. Ligadura y sección del cayado de la vena ácidos. D. Bocio ya liberado de las estructuras mediastinales.

resección completa del bocio y requiriendo en el $10 \%$ restante otros abordajes ya sean aislados o en combinación con la cervicotomía $(\underline{1}, \underline{9})$.

La esternotomía es necesaria en un 2-5\% de los pacientes y suele utilizarse para la resección de bocios de gran tamaño que no puedan extraerse por abordaje cervical, bocios con irrigación torácica, bocios en mediastino posterior, con extensión al arco aórtico, que causen síndrome de vena cava superior, recurrentes, bocios malignos con o sin metástasis, o en pacientes con tiroidectomía cervical previa o bocio primario (10-12).

Algunos grupos quirúrgicos utilizan los criterios de Katlic para definir la necesidad de un abordaje extracervical: si por tomografía más del 50 \% del bocio es subesternal se anticipa que pueda requerir la realización de una esternotomía $(\underline{1}, \underline{13}, 14)$.

A pesar que la esternotomía es un abordaje efectivo que permite excelente visualización del bocio y del área mediastinal, hay que tener en cuenta las posibles complicaciones asociadas como el dolor postoperatorio, complicaciones pulmonares, dehiscencia esternal, mediastinitis y molestias por el material de sutura, entre otras $(\underline{15}, \underline{16})$. 
Sin embargo, y según la literatura disponible, es válido proponer la videotoracoscopia como una técnica segura y eficaz, con baja morbimortalidad, para el tratamiento quirúrgico del bocio intratorácico.
Rolighed et al. en una serie de 52 pacientes reportan $21 \%$ de complicaciones pulmonares, lo cual prolongó significativamente la estancia hospitalaria en pacientes con abordaje por esternotomía (14).

La toracotomía es otro abordaje extracervical que permite acceder al mediastino posterior y medio $(\underline{5}, 17,18)$. Kacprazk et al. y Ojanguren et al., reportan uno y dos casos respectivamente de resección de un bocio intratoracico por abordaje combinado de cervicotomía mas toracotomía derecha. Los autores hacen énfasis en que esta técnica combinada ofrece una amplia exposición, facilita la resección de la masa y reduce complicaciones catastróficas como sangrado masivo (4;프).

La esternotomía es superior a la toracotomía para visualizar el mediastino anterior, pero la toracotomía ofrece una mejor visión del mediastino medio y posterior, principalmente si hay extensión del bocio por debajo del arco aórtico y/o compresión de la tráquea $(5,18)$.

La utilización de la cirugía robótica para el abordaje del mediastino por el hemitórax derecho más cervicotomía también ha sido descrita, con resultados similares a los obtenidos por videotoracoscopia $(\underline{19}, \underline{20})$. Recientemente Gupta et al. hacen una revisión retrospectiva de su experiencia, presentando siete casos de resección de bocio intratoracico por videotoracoscopia más cervicotomía, con adecuado control del dolor, una estancia media hospitalaria de cinco días, baja morbilidad y ningún caso de muerte asociada (21).

Por su parte, Grondin et al. reportan un caso de bocio intratorácico con resección quirúrgica por toracoscopia más cervicotomía sin complicaciones asociadas (22). Más recientemente Arango et al. describen el abordaje por videotoracoscopia por único puerto de un bocio intratorácico primario (23).

Hasta nuestro conocimiento estos son los únicos reportes en la literatura en los cuales la videotoracoscopia hace parte de la técnica quirúrgica para el abordaje del bocio intratorácico, por lo que es necesario realizar más estudios para poder tener resultados con mayor peso estadístico y poder comparar la videotoracoscopia con otras técnicas como la toracotomía y la esternotomía; sin embargo, y según la literatura disponible, es válido proponer la videotoracoscopia como una técnica segura y eficaz, con baja morbimortalidad, para el tratamiento quirúrgico del bocio intratorácico.

En el caso clínico descrito, dadas las características de la masa y su localización en el mediastino posterior, la adecuada exposición del mediastino por videotoracoscopia, sumado a las ventajas ampliamente conocidas de la cirugía mínimamente invasiva con respecto al control del dolor, estancia hospitalaria, días de drenaje pleural y menor agresión tisular (24-25), se decidió remplazar la toracotomía por la videotoracoscopia derecha con una buena exposición y liberación del bocio, sin presentarse ninguna complicación, con control del dolor, con buen tiempo de drenaje pleural y una estancia hospitalaria dentro de lo esperado con respecto a la literatura.

\section{Conclusión}

La combinación de cervicotomía con videotoracoscopia permite la intervención de pacientes seleccionados con bocio intratorácico medio/posterior de forma segura y efectiva, sumándole las ventajas de la cirugía mínimamente invasiva en cuanto a la morbilidad, estancia hospitalaria y pronta recuperación. 


\section{Conflicto de intereses}

Los autores declaran no tener conflicto de intereses.

\section{Bibiliografía}

1. Rios A, Sitges-Serra A. Surgical treatment of intrathoracic goitre. Cir Esp 2012 Aug;90(7):421-8. https://www.ncbi.nlm.nih.gov/pubmed/22464974

2. Hegedus L, Bonnema SJ. Approach to management of the patient with primary or secondary intrathoracic goiter. J Clin Endocrinol Metab 2010 Dec;95(12):515562. https://www.ncbi.nlm.nih.gov/pubmed/21131536

3. Sakkary MA, Abdelrahman AM, Mostafa AM, Abbas AA, Zedan MH. Retrosternal goiter: the need for thoracic approach based on CT findings: surgeon's view. J Egypt Natl Canc Inst 2012 Jun;24(2):85-90. https://www.ncbi.nlm.nih.gov/pubmed?linkname $=$ pubmed pubmed\&from uid $=17416216$

4. Ojanguren AA, Baena Fustegueras JA, Ros LS, Santamaría GM, Ojanguren A, I, OLsina Kissle JJ. Best approach for posterior mediastinal goiter removal: transcervical incision and lateral thoracotomy. Arch Bronconeumol 2014 Jun;50(6):255-7. http://www.archbronconeumol.org/es/

5. Kacprzak G, Karas J, Rzechonek A, Blasiak P. Retrosternal goiter located in the mediastinum: surgical approach and operative difficulties. Interact Cardiovasc Thorac Surg 2012 Nov;15(5):935-7. https://www.ncbi.nlm.nih.gov/pub$\underline{\mathrm{med} / 22922389}$

6. Gao B, Jiang Y, Zhang X, Zhao J, He Y, Wen Y, et al. Surgical treatment of large substernal thyroid goiter: analysis of 12 patients. Int J Clin Exp Med 2013;6(7):488-96. https://www.ncbi.nlm.nih.gov/pmc/articles/PMC4709748/

7. Kanzaki R, Higashiyama M, Oda K, Okami J, Maeda J, Takenaka A, et al. Surgical management of primary intrathoracic goiters. Gen Thorac Cardiovasc Surg 2012 Mar;60(3):171-4. http://www.springer.com/medicine/surgery/journal/11748

8. De Aguiar-Quevedo K, Ceron-Navarro J, Jorda-Aragon C, Pastor-Martinez E, Sales-Badia JG, Garcia-Zarza A, et al. [Intrathoracic goitre: a literature review]. Cir Esp 2010 Sep;88(3):142-5. http://www.elsevier.es/es-revista-cirugia-espanola-36-articulo-bocio-intratoracico-revision-literatura-medica-S0009739X1000134X

9. Rios A, Rodriguez JM, Febrero B, Balsalobre MD, Tebar FJ, Parrilla P. Toxic intrathoracic goiter. Clinical profile and surgical morbidity in an endocrine surgery unit. Endocrinol Nutr 2010 May;57(5):196-202.

10. Komninos G, Galata' G, Schulte KM. Giant recurrent intrathoracic goitre treated by clamshell thoracotomy and reverse sternotomy. BMJ Case Rep 2014;2014. http://casereports.bmj.com/content/2014/bcr-2013-202790.abstract

11. Scognamillo F, Attene F, Paliogiannis P, Ruggiu MW, Cossu A, Trignano M. Is sternotomy always necessary for the treatment of mediastinal ectopic thyroid goiter? Ann Ital Chir 2014 May;85(3):304-7. https://www.ncbi.nlm.nih.gov/pubmed/23899670 
12. Cohen JP. Substernal goiters and sternotomy. Laryngoscope 2009 Apr;119(4):683-8. pubmed

13. Coskun A, Yildirim M, Erkan N. Substernal goiter: when is a sternotomy required? Int Surg 2014 Jul;99(4):419-25. https://www.ncbi.nlm.nih.gov/pubmed/25058777

14. Rolighed L, Ronning H, Christiansen P. Sternotomy for substernal goiter: retrospective study of 52 operations. Langenbecks Arch Surg 2015 Apr;400(3):301-6. http://link.springer.com/article/10.1007/s00423-015-1288-9

15. Randolph GW, Shin JJ, Grillo HC, Mathisen D, Katlic MR, Kamani D, et al. The surgical management of goiter: Part II. Surgical treatment and results. Laryngoscope 2011 Jan;121(1):68-76.

16. Casella C, Pata G, Cappelli C, Salerni B. Preoperative predictors of sternotomy need in mediastinal goiter management. Head Neck 2010 Sep;32(9):1131-5. https://www.ncbi.nlm.nih.gov/pubmed/19953632

17. Nistor C, Ciuche A, Motas C, Motas N, Bluoss C, Pantile D, et al. Cervico-mediastinal thyroid masses - our experience. Chirurgia (Bucur ) 2014 Jan;109(1):34-43 https://www.ncbi.nlm.nih.gov/pubmed/24524468

18. Machado NO, Grant CS, Sharma AK, al Sabti HA, Kolidyan SV. Large posterior mediastinal retrosternal goiter managed by a transcervical and lateral thoracotomy approach. Gen Thorac Cardiovasc Surg 2011 Jul;59(7):507-11. https://www.ncbi. nlm.nih.gov/pubmed/21751115

19. Wang S, Xu S, Liu B. Resection of huge retrosternal goiter through a novel combined cervical and robot-assisted approach. Artif Organs 2014 May;38(5):431-3. https://www.ncbi.nlm.nih.gov/pubmed/24117750

20. Al-Mufarrej F, Margolis M, Tempesta B, Strother E, Gharagozloo F. Novel thoracoscopic approach to posterior mediastinal goiters: report of two cases. J Cardiothorac Surg 2008;3:55. https://www.ncbi.nlm.nih.gov/pmc/articles/PMC2577642/

21. Gupta P, Lau KK, Rizvi I, Rathinam S, Waller DA. Video assisted thoracoscopic thyroidectomy for retrosternal goitre. Ann R Coll Surg Engl 2014 Nov;96(8):6068. https://www.ncbi.nlm.nih.gov/pubmed/25350184

22. Grondin SC, Buenaventura P, Luketich JD. Thoracoscopic resection of an ectopic intrathoracic goiter. Ann Thorac Surg 2001 May;71(5):1697-8. pubmed

23. Arango TE, Baamonde LC, Algar Algar FJ, Salvatierra VA. Single-port thoracoscopic access for a mediastinal ectopic goiter. Cir Esp 2015 Apr;93(4):264-6.

24. Nakajima J, Takamoto S, Kohno T, Ohtsuka T. Costs of videothoracoscopic surgery versus open resection for patients with of lung carcinoma. Cancer $2000 \mathrm{Dec}$ 1:89(11 Suppl):2497-501. https://www.ncbi.nlm.nih.gov/pubmed/11147635

25. Fournel L, Zaimi R, Grigoroiu M, Stern JB, Gossot D. Totally thoracoscopic major pulmonary resections: an analysis of perioperative complications. Ann Thorac Surg 2014 Feb;97(2):419-24. https://www.ncbi.nlm.nih.gov/pubmed/24266953 